\title{
Influencia del tamaño del cristal de cinc de láminas de acero galvanizadas en ambientes que contienen cloruros y sulfotos
}

\author{
Zinc galvanized steel sheets crustal size influence in environments \\ containing chlorides and sulfates
}

Influência do tamanho do cristal de zinco de lâminas de aço
galvanizadas em ambientes que contêm doretos e sulfatos

Fecha de Recepción: 22 de Octubre de 2014

Fecha de Aceptación: 08 de Abril de 2015

\author{
Julieth Andrea Ramírez-Jerez* \\ Mario Valero-Alvarado** \\ Darío Yesid Peña-Ballesteros*** \\ Jhon Edisson Torres-Ramírez ${ }^{\star * * *}$
}

\section{Resumen}

El trabajo evaluó la influencia del tamaño del cristal de cinc en el comportamiento electroquímico de láminas de acero galvanizadas por inmersión en caliente. Los ensayos se realizaron en una celda electroquímica plana

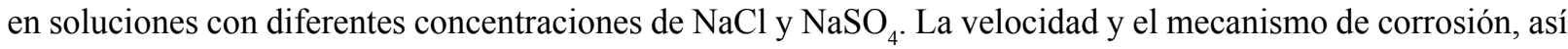
como las características resistivas y difusivas de las láminas se evaluaron mediante técnicas electroquímicas, como espectroscopia de impedancia electroquímica (EIS), resistencia a la polarización (Rp) y curvas potenciodinámicas. La composición y distribución de los productos de corrosión (FeO y algunos compuestos de cinc) y el daño superficial causado por estos se determinaron por medio de DRX y SEM-EDS. Los resultados mostraron que la degradación de la capa de óxidos formada se ve afectada por la presencia de cloruros que penetran, llegan a la superficie del metal base y generan corrosión localizada.

Palabras clave: Acero galvanizado, Cristales de cinc, Corrosión.

\footnotetext{
* Universidad Industrial de Santander (Bucaramanga - Santander, Colombia). juliethramirezj@gmail.com.

** Universidad Industrial de Santander (Bucaramanga - Santander, Colombia). mariovalero23@gmail.com.

*** Ph.D. Universidad Industrial de Santander (Bucaramanga - Santander, Colombia).dypena@uis.edu.co.

**** Universidad Industrial de Santander (Bucaramanga - Santander, Colombia). jtorram@gmail.com.
} 


\section{Abstract}

This paper studied the influence of the zinc crystal size in the electrochemical behavior of steel sheets hot-dip galvanized. The tests were performed in an electrochemical flat cell in solutions with different concentrations of $\mathrm{NaCl}$ and $\mathrm{NaSO}_{4}$. The rate, mechanism of corrosion and the resistive and diffusive characteristics of the sheets were tested by electrochemical techniques like electrochemical impedance spectroscopy (EIS), polarization resistance (Rp) and potentiodynamic polarization curves. The composition and distribution of the products of corrosion (FeO and some zinc compounds) and the surface damage caused by them, were determined using XRD and SEM-EDS. Results showed that the degradation of the oxide layer formed is affected by the presence of chlorides, which penetrate reaching the surface of the base metal, producing localized corrosion.

Keywords: Galvanized steel, corrosion, chlorides, sulphates, resistance polarization, electrochemical impedance spectroscopy (EIS), potentiodynamic curves.

\section{Resumo}

O trabalho avaliou a influência do tamanho do cristal de zinco no comportamento eletroquímico de lâminas de aço galvanizadas por imersão a quente. Os ensaios se realizaram em uma câmara eletroquímica plana em soluções com diferentes concentrações de $\mathrm{NaCl}$ e $\mathrm{NaSO}_{4}$. A velocidade e o mecanismo de corrosão, assim como as características resistivas e difusivas das lâminas se avaliaram mediante técnicas eletroquímicas, como espectroscopia de impedância eletroquímica (EIS), resistência à polarização $(\mathrm{Rp})$ e curvas potenciodinâmicas. A composição e distribuição dos produtos de corrosão ( $\mathrm{FeO}$ e alguns compostos de zinco) e o dano superficial causado por estes se determinaram por meio de DRX e SEM-EDS. Os resultados mostraram que a degradação da capa de óxidos formada se vê afetada pela presença de cloretos que penetram, chegam à superfície do metal base e geram corrosão localizada.

Palavras chave: Aço galvanizado, Cristais de zinco, Corrosão.

Cómo citar este artículo:

[1] J.A. Ramírez-Jerez, M. Valero-Alvarado, D.Y. Peña-Ballesteros, J.E. Torres-Ramírez, "Influencia del tamaño del cristal de cinc de láminas de acero galvanizadas en ambientes que contienen cloruros y sulfatos", Fac. Ing., vol. 24 (39), pp. 99-108, Mayo-Ago. 2015. 


\section{INTRODUCCIÓN}

Durante los últimos años se ha implementado el galvanizado por inmersión en caliente como método de protección catódica para contrarrestar la degradación del acero. Debido a que el cinc es un metal más activo que el hierro, genera una película protectora en la superficie que prolonga la vida media del recubrimiento; sin embargo, esta película, al estar en contacto con medios corrosivos industriales con alta humedad o salinidad costera, disminuye la resistencia a la corrosión de la lámina de acero galvanizado. En pro de mejorar las propiedades del recubrimiento, se han determinado factores que afectan el proceso electroquímico de las láminas de acero galvanizadas, tales como el espesor de la capa de cinc, la concentración de cloruros y sulfatos presentes en el medio corrosivo, la concentración de productos de corrosión y la condición superficial del material.

El estudio que aquí se presenta se realizó con el fin de observar el comportamiento sinérgico de diferentes tamaños del cristal de cinc, inmerso en concentraciones de cloruros y sulfatos, $\mathrm{y}$, además, de evaluar la influencia de dichos cristales formados por el proceso de galvanizado por inmersión en caliente, como posible factor determinante en la velocidad de corrosión.

El acero galvanizado es aquel que se obtiene luego de un proceso de recubrimiento de cinc sobre el acero. Por lo general, el galvanizado por inmersión en caliente es el más empleado en la actualidad, consiste en hacer una inmersión de la pieza en un baño de cinc fundido, a una temperatura de $450^{\circ} \mathrm{C}$.

El recubrimiento de cinc formado en la superficie, se caracteriza por la formación de capas de aleación Fe-Zn, cuya composición de Zn varía a partir de la superficie del acero, denominadas fase gamma $(\Gamma)$, delta $(\delta)$, zeta $(\zeta)$ y fase externa eta $(\eta)$; esta última capa, que solo contiene cinc, le confiere al recubrimiento su aspecto característico gris metálico brillante [1].

\section{Cristal de cinc de galvanizado}

Los cristales de cinc se forman en la superficie de la lámina galvanizada en continuo por inmersión en caliente; se presentan generalmente en dos tamaños: normal, es decir, cristales de tamaño ordinario (15-25 $\mathrm{mm})$ que cubren la superficie completa de la lámina, y minimizado (o mínimo), cuya dimensión es más pequeña $(1 \mathrm{~mm})$ y uniforme [2].

\section{Proceso de solidificación de los cristales de cinc}

Los cristales de cinc se producen por la solidificación del cinc fundido, que se adhiere a la plancha de acero $\left(419{ }^{\circ} \mathrm{C}\right)$. Los cristales que se forman tienen una simetría hexagonal. Cuando el recubrimiento está completamente solidificado, se definen granos específicos de cinc. Una alta velocidad de nucleación durante el proceso de solidificación causa la formación de granos pequeños en la estructura solidificada final, mientras que una baja velocidad favorece el crecimiento de granos grandes [2,3].

Los cristales de cinc también pueden incrementar su tamaño debido a agregados de elementos aleantes o impurezas en el metal fundido; por ejemplo, el plomo es una impureza común en el cinc, pues se encuentra presente en los minerales que contienen $\mathrm{Zn}$; el plomo se precipita en la superficie del recubrimiento, y la distribución de las partículas de plomo a lo ancho de la superficie define la apariencia visible (cristales opacos o cristales brillantes). En la actualidad existen diversas preocupaciones ambientales por el uso del plomo, razón por la cual se ha implementado el uso de pequeñas cantidades de antimonio $(0.03$ y $0.10 \%)$ al baño de cinc, dando como resultado un recubrimiento liso $[9,12,13]$.

Los procesos de galvanizado por inmersión adicionan pequeñas cantidades de plomo o antimonio en el baño de cinc para disminuir la tensión superficial, mejorar la adherencia del recubrimiento y obtener superficies más uniformes; por otra parte, estos elementos se nuclean en los cristales de cinc y forman diferentes morfologías, denominadas floreados [4]. Para caracterizar los diferentes tamaños del cristal de cinc formados, se realizó un análisis mediante microscopía óptica que estableció la relación en cantidad y tamaño de los cristales opacos y brillantes característicos del galvanizado [13]. 


\section{DESCRIPCIÓN DEL TRABAJO}

\section{A. Materiales}

Por medio de un análisis de composición elemental, por espectrometría de emisión óptica, en un espectrómetro
Bruker de referencia Q4 Tasman, se determinaron los elementos presentes en el metal base, dando como resultado un acero de bajo carbono, como se muestra en la Tabla 1.

TABLA 1

COMPOSICIÓN ELEMENTAL DEL METAL BASE

\begin{tabular}{|c|c|c|c|c|c|c|c|}
\hline Elemento & $\mathrm{Fe}$ & $\mathrm{C}$ & $\mathrm{Si}$ & $\mathrm{Mn}$ & $\mathrm{S}$ & $\mathrm{Ni}$ & $\mathrm{Cu}$ \\
\hline$\%$ & 97.16 & 0.104 & 0.029 & 0.287 & $<0.150$ & 0.316 & 1.050 \\
\hline
\end{tabular}

La medición del espesor del recubrimiento de cinc (22 $\mu \mathrm{m})$ se realizó teniendo en cuenta la norma ASTM E-376. Las probetas usadas de acero galvanizadas por inmersión en caliente se prepararon con un tamaño de $2 \times 2 \mathrm{~cm}$., y fueron montadas en una celda electroquímica plana, según la norma ASTM G-109, la cual consistía de un electrodo de referencia plata/ cloruro de plata, un electrodo auxiliar de grafito de alta pureza y las probetas de acero galvanizado como electrodos de trabajo. El electrolito usado para las pruebas de inmersión variaba en concentración de $\mathrm{Cl}^{-}$ y $\mathrm{SO}_{4}^{-}$, como se muestra en la Tabla 2.

TABLA 2

Concentraciones de Cl- y SO4- Del electrolito

\begin{tabular}{|c|c|c|c|c|c|c|c|c|}
\hline \multirow{2}{*}{ Reactivos } & \multirow{2}{*}{ Unidades } & \multicolumn{7}{|c|}{ Soluciones de trabajo } \\
\cline { 3 - 10 } & & $\mathbf{1}$ & $\mathbf{2}$ & $\mathbf{3}$ & $\mathbf{4}$ & $\mathbf{5}$ & $\mathbf{6}$ & $\mathbf{7}$ \\
\hline \multirow{2}{*}{$\mathrm{NaCl}$} & \multirow{2}{*}{$\mathrm{g} / 400 \mathrm{~L}$} & 4.33 & 15.15 & 4.33 & 15.15 & 9.74 & 9.74 & - \\
\cline { 3 - 10 } & & 5.36 & 5.36 & 16.08 & 16.08 & 10.72 & - & 10.72 \\
\hline $\mathrm{Na}_{2} \mathrm{SO}_{4}$ & & &
\end{tabular}

\section{B. Pruebas electroquímicas}

Las muestras fueron inicialmente inmersas en un baño ultrasónico con etanol, durante un período de $10 \mathrm{~min}$., aproximadamente, con el fin de retirar las impurezas presentes en la superficie del recubrimiento. Las técnicas electroquímicas empleadas fueron potencial de circuito abierto (OCP), por $3600 \mathrm{~s}$; espectroscopia de impedancia electroquímica $(E I S)$, tomando valores de frecuencia inicial y final de 100.000 y $0.05 \mathrm{~Hz}$, respectivamente; resistencia a la polarización lineal (Rp), con una velocidad de barrido de $0.125 \mathrm{mV} / \mathrm{s}$, y polarización potenciodinámica (PDP), con una frecuencia desde -0.5 hasta $1.8 \mathrm{~V}$ respecto al OCP, con un barrido de $1.6 \mathrm{mV} / \mathrm{s}$, en un potenciostato/ galvanostato GAMRY 750. Las muestras de trabajo que se usaron tenían un tamaño del cristal, o tamaño del floreado, grande (FG) y pequeño (FP).

\section{Estudio morfológico}

Para determinar los productos de corrosión formados sobre las muestras de acero galvanizadas, y el tipo de daño superficial que se presentó, se realizaron pruebas de microscopia electrónica de barrido (SEM) con espectrómetro de energía dispersiva $(E D S)$ y difracción de rayos $\mathrm{X}(D R X)$.

\section{Resultados}

\section{A. Curvas de polarización potenciodinámicas}

Con el fin de evaluar la influencia del tamaño del floreado o del cristal (pequeño y grande) en el comportamiento electroquímico de láminas de acero galvanizadas, se parte del análisis de las curvas potenciodinámicas, como se muestran en la Fig. 1. 

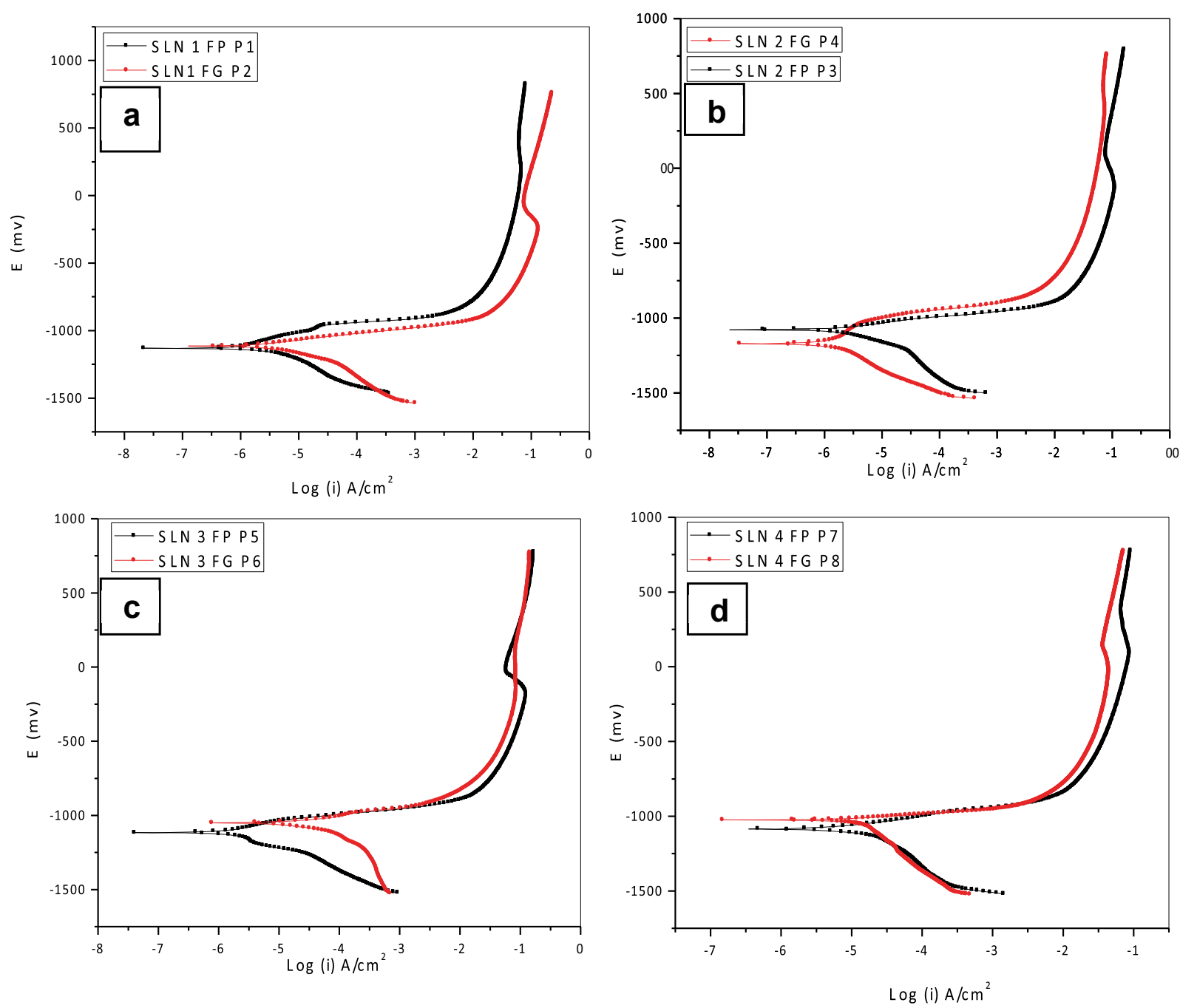

Fig. 1. Curvas de polarización potenciodinámicas de acero galvanizado para tamaño del cristal pequeño y grande. (a) Solución 1, (b) Solución 2, (c) Solución 3, (d) Solución 4.

En las figuras 1(a), 1(c) y 1(d) se observa un desplazamiento de la densidad de corriente hacia valores más positivos, indicando un mayor deterioro del recubrimiento; esto se debe, por una parte, a que las concentraciones de los iones agresivos interactúan con la superficie del recubrimiento. Haciendo una comparación entre la solución 1 y la 2 , se espera que a altos contenidos de iones cloruro, el deterioro del recubrimiento sea mayor, pero por medio de los resultados obtenidos se puede observar que se presenta mayor velocidad de corrosión para la solución 1 , como consecuencia del efecto de los iones de cloruro, que inician el ataque por difusión a través de la capa de galvanizado, llegando al sustrato o material base; pero son los sulfatos los encargados del deterioro de este, debido, principalmente, a la formación de compuestos solubles que se disuelven en el electrolito y se reabsorben en la superficie del galvanizado, atacando el sustrato; este proceso ocurre de manera repetitiva [5].

Por otra parte, también se puede observar la influencia que ejerce el tamaño del cristal en el proceso corrosivo del galvanizado. En las figuras 1(a), 1(c) y 1(d) se puede observar que los cristales grandes presentan mayor velocidad de corrosión que los cristales pequeños. Cabe destacar que para la solución 3 , con alto contenido de sulfatos, la velocidad de corrosión aumentó considerablemente en relación con las otras soluciones [6]. 
En las curvas potenciodinámicas se evidencia la influencia del tamaño del cristal de cinc en la velocidad de corrosión de las láminas de acero galvanizadas por inmersión en caliente, expuestas a diferentes concentraciones de cloruros y sulfatos. Las velocidades de corrosión calculadas para las diferentes soluciones se basaron en la norma ASTM G102, y los resultados se pueden observar en la Tabla 3.

\section{TABLA 3}

VELOCIDADES DE CORROSIÓN

\begin{tabular}{|c|c|c|c|c|}
\hline \multirow{2}{*}{ SOLUCIÓN } & $\begin{array}{c}\text { Tamaño del } \\
\text { cristal }\end{array}$ & $\begin{array}{c}\mathbf{E}_{\text {corr }} \\
(\mathbf{m v})\end{array}$ & $\begin{array}{c}\mathbf{I}_{\text {corr }} \\
\boldsymbol{\mu A} / \mathbf{c m}^{2}\end{array}$ & $\mathbf{m p y}$ \\
\hline \multirow{2}{*}{1} & Pequeño & -1130 & 2,0788 & 0,950 \\
\cline { 2 - 5 } & Grande & -1114 & 2,7249 & 1,245 \\
\hline \multirow{2}{*}{2} & Pequeño & -1079 & 1,4387 & 0,657 \\
\cline { 2 - 5 } & Grande & -1171 & 1,0782 & 0,484 \\
\hline \multirow{2}{*}{3} & Pequeño & -1099 & 2,0716 & 0,945 \\
\cline { 2 - 5 } & Grande & -1050 & 12,9552 & 5,919 \\
\hline \multirow{2}{*}{4} & Pequeño & -1086 & 8,3550 & 3,818 \\
\cline { 2 - 5 } & Grande & -1025 & 12,6220 & 5,767 \\
\hline
\end{tabular}

\section{B. Espectroscopia de impedancia electroquímica (EIS)}

Con el fin de estudiar las características resistivas y capacitivas del recubrimiento, se llevó a cabo un análisis mediante EIS. Por medio de los diagramas de Nyquist (ver figuras 2(a), 2(c) y 2(d)) se puede observar que la resistencia a la polarización de los cristales grandes es menor en las soluciones 1, 3 y 4; por esta razón se confirma que la velocidad de corrosión es mayor. Además, estos resultados se pueden relacionar con las características morfológicas de los cristales de cinc, pues los cristales pequeños presentan un comportamiento más resistivo que los floreados grandes, debido a que son más homogéneos en la superficie, dado que su crecimiento es paralelo a la superficie del galvanizado e impide que se depositen impurezas, a diferencia del floreado grande, que presenta una estructura porosa, es decir, con alto valor de capacitancia. El comportamiento del diagrama de Nyquist para la solución 2 presenta un comportamiento contrario a los mencionados anteriormente, debido a que la flor grande es la que presenta mayor resistencia a la polarización, por lo tanto, menor velocidad de corrosión; esto se atribuye a que la zona expuesta $\left(1 \mathrm{~cm}^{2}\right)$ tomó zonas brillantes de la flor grande, favoreciendo la resistencia a la corrosión. 

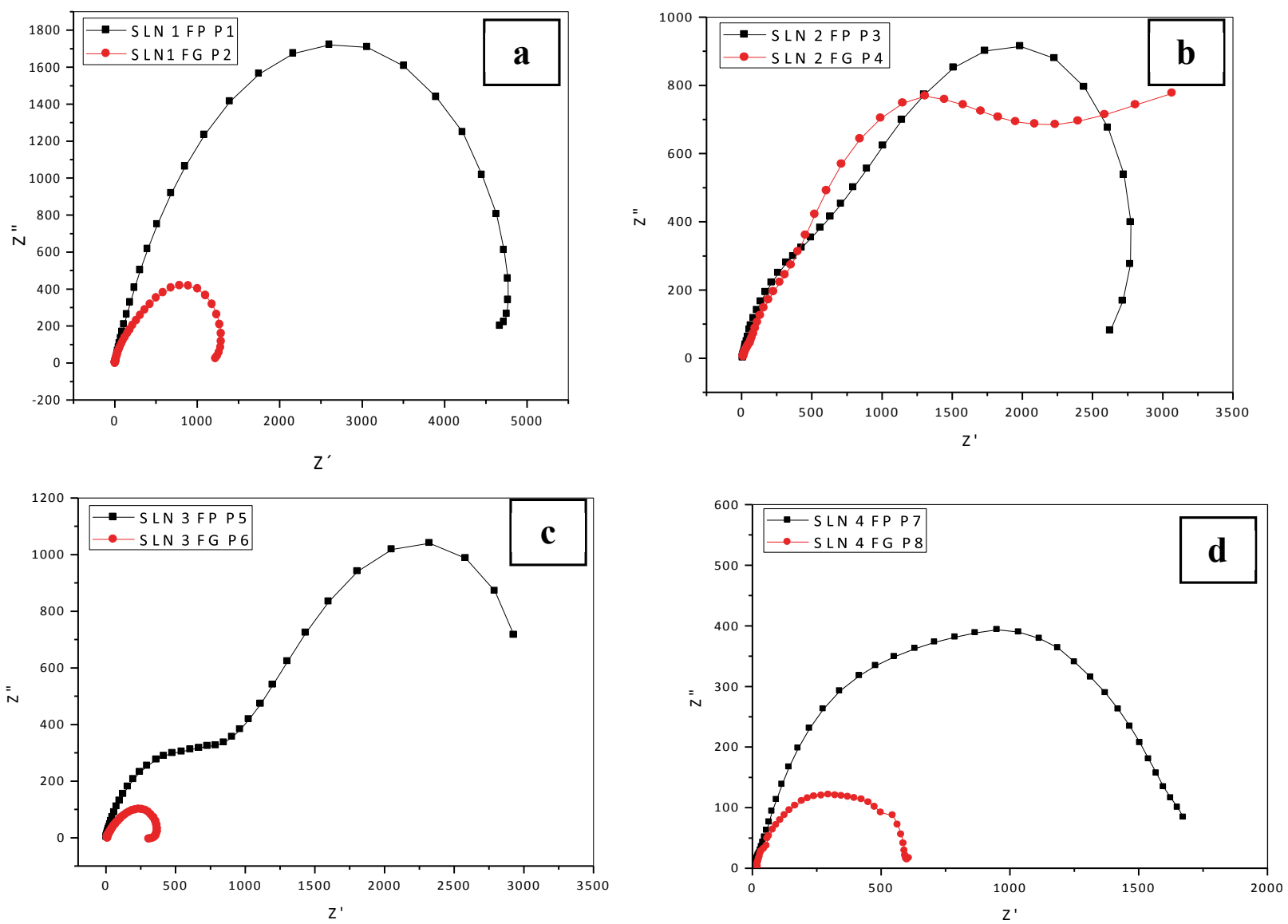

Fig. 2. Comparación curvas de Nyquist de acero galvanizado para tamaño de flor pequeña y grande. (a) Solución 1, (b) Solución 2, (c) Solución 3, (d) Solución 4.

\section{DRX Y SEM}

Mediante microscopia electrónica de barrido fue posible identificar la presencia de diferentes morfologías en la superficie del recubrimiento. En la Fig. 3a se observa la formación de islas asociadas a cristales pseudo-hexagonales que se ubican en la superficie del galvanizado [7]; dichos cristales, inicialmente, se asociaban a productos de corrosión comunes, como la simonkolleita $\left(\mathrm{Zn}_{5}(\mathrm{OH})_{8} \mathrm{Cl}_{2}\right.$ $\left(\mathrm{H}_{2} \mathrm{O}\right)$ ), formados principalmente por la presencia de iones cloruro, pero los resultados de EDS indican que el compuesto formado corresponde a la Gordaita $\left(\mathrm{Zn}_{4} \mathrm{Na}(\mathrm{OH})_{6}\left(\mathrm{SO}_{4}\right) \mathrm{Cl}\left(\mathrm{H}_{2} \mathrm{O}\right)_{6}\right)$; esto se debe a la evolución de una fase a otra, por la presencia de sulfatos. Para este caso, según Castaño [9], se lleva a cabo una transformación morfológica; inicialmente, el carbonato de cinc pasa a ser simonkolleita, y una parte de esta, a gordaita, como producto final de corrosión que nuclea en los defectos de manera ortogonal a la superficie del recubrimiento $[8,9]$.

Por otra parte, en esta misma micrografía, donde se señala la zona más clara, se puede observar mediante EDS la presencia de altos picos de hierro, que junto a las pequeñas cantidades de azufre, oxígeno y carbono dan, posiblemente, paso a la formación de hierro, denominado Hohmannita $\mathrm{Fe}_{2}\left(\mathrm{H}_{2} \mathrm{O}\right)_{4}\left(\left(\mathrm{SO}_{4}\right)_{2} \mathrm{O}\right)\left(\mathrm{H}_{2} \mathrm{O}\right)_{4}$. 

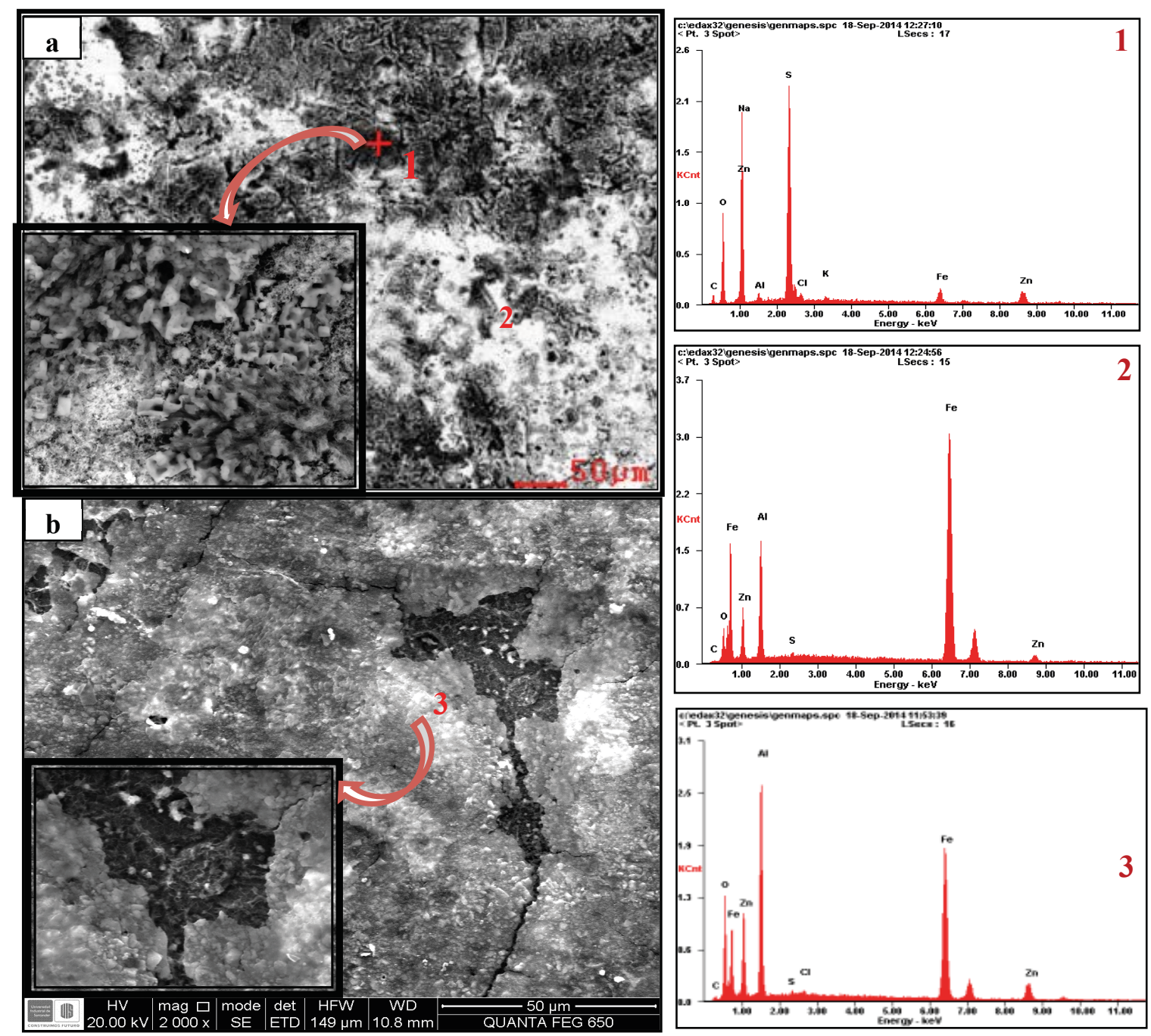

Fig. 3. Micrografías de SEM y EDS para diferentes tamaños de flor en solución 4. [0,64 M NaCl y 0,28 M de Na2SO4]. a) Flor pequeña. b) Flor grande.

En los procesos de galvanizado por inmersión en caliente se adicionan algunos elementos al baño del recubrimiento de cinc, como plomo, estaño, cobre, níquel y aluminio, siendo este último uno de los elementos más importantes en el proceso, ya que no solo mejora la resistencia a la corrosión y aumenta el brillo del revestimiento, sino que asegura una buena adherencia del recubrimiento en el sustrato y le proporciona ductilidad [10].

Según la Asociación Latinoamericana de Cinc LATIZA- [11], las cantidades de aluminio que debe contener el baño de cinc están entre 0,15 y $0,17 \%$, pero al analizar posteriormente la superficie del galvanizado se observa un incremento en la cantidad de este elemento (entre 0.25 y $0,40 \%$ ); esto se debe a la fuerte afinidad que tiene el aluminio con el hierro, formando una capa de inhibición $\mathrm{Fe}_{2} \mathrm{Al}_{5}$ que retarda, pero no eliminan la reacción de compuestos intermetálicos frágiles Fe-Zn.

Las figuras $3 \mathrm{a}$ y $3 \mathrm{~b}$ muestran una cantidad representativa tanto de aluminio como de hierro, que probablemente reaccionaron para formar un compuesto intermetálico de $\mathrm{Fe}-\mathrm{Al}$ que mejora la resistencia del recubrimiento a la corrosión; como se mencionó, este compuesto se caracteriza por ubicarse entre el sustrato y el recubrimiento del cinc. Debido a que estas micrografías corresponden a las muestras atacadas con la solución 4 , las altas concentraciones 
de cloruros y sulfatos deterioraron los primeros productos de corrosión formados en los floreados grandes, permitiendo de esta manera observar la capa de Fe-Al formada.

También se puede observar que a diferencia de la Fig. $3 \mathrm{a}$ (floreado pequeño), la figura $3 \mathrm{~b}$ muestra un mayor deterioro del recubrimiento, lo que se puede atribuir, principalmente, a la agresividad de la solución y al tamaño del cristal del cinc. De esta manera, se puede afirmar que las muestras con floreado grande presentan un efecto perjudicial en la resistencia a la corrosión; esto se debe a que en el proceso de galvanizado se adicionan elementos como antimonio y plomo, que disminuyen los sitios de nucleación de planos basales
(00.2), que se caracterizan por tener superficies más lisas, y favorecen los sitios de nucleación de planos piramidales (20.1), donde el revestimiento se hace más débil en cuanto a los ataques por corrosión, ya que estos planos son más activos electroquímicamente y requieren de menor energía para romper estos enlaces [6].

Según los resultados del DRX, en la Fig. 4 se pueden observar las fases cristalinas formadas, como la Gordaita $(\mathrm{Zn} 4 \mathrm{Na}(\mathrm{OH}) 6$ (SO4)Cl( $\mathrm{H} 2 \mathrm{O}) 6)$, como producto final de corrosión que nuclea en los defectos de manera ortogonal a la superficie del recubrimiento, como se mencionó anteriormente, afectando en mayor proporción el galvanizado.

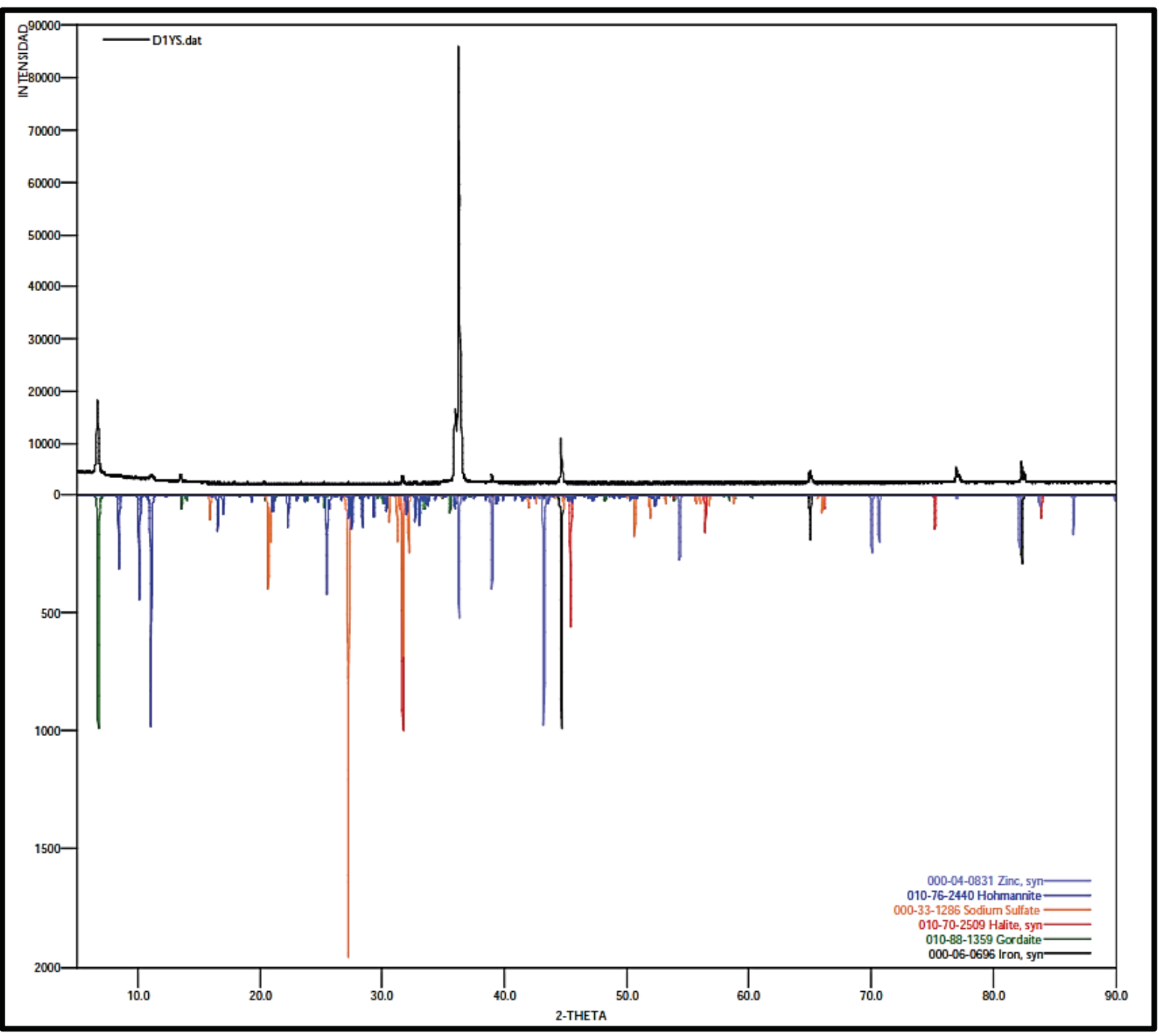

Fig. 4. Difracción de rayos x para la solución 4 flor grande 


\section{Conclusiones}

Dado que se determinó que el tamaño de flor es un factor determinante en la velocidad de corrosión, se recomienda trabajar con tamaños de floreado pequeño, ya que forman planos basales que crecen paralelos a la superficie del recubrimiento, dificultando la deposición de impurezas que pueden deteriorar el recubrimiento, $\mathrm{y}$, por ende, disminuir la resistencia a la corrosión de estructuras galvanizadas.

Los estudios indican que el tamaño de floreado grande tiene un efecto perjudicial en la resistencia a la corrosión, debido a la nucleación de planos piramidales, que son más activos electroquímicamente, es decir, tiene una energía de enlace es muy baja; por esta razón, se requiere de menor energía para romper estos enlaces. Por otra parte, el crecimiento ortogonal a la superficie del galvanizado de dichos planos favorece la deposición de especies agresivas que pueden llegar a difundir, deteriorando el sustrato.

La muestra que presentó menor resistencia a la corrosión corresponde a un tamaño de flor grande, expuesta a concentraciones con bajo contenido de cloruro y alto contenido de sulfatos. Esto se debe a que los floreados grandes del galvanizado tienen una estructura piramidal, donde se posan preferencialmente los sulfatos. Lo anterior se asocia al comportamiento inductivo que se hizo evidente en el diagrama de Nyquist a bajas frecuencias, presentándose una reabsorción de especies en la superficie por parte de los iones de sulfato.

\section{REFERENCIAS}

[1] A. P. Yadav, H. Katayama A., K. Noda A., H. Masuda A., A. Nishikata B., T. TSURU. "Effect of $\mathrm{Fe}-\mathrm{Zn}$ alloy layer on the corrosion resistance of galvanized steel in chloride containing environments", Corrosion Science 49 (2007), 3716-3731.

[2] Asociación Latinoamericana de Cinc-LATIZA-. GalvinfoNotes, Proceso de Recubrimiento y Tratamiento de Superficies. 2008.

[3] ASTM A 653/A 653 M. Especificación para la Plancha de Acero Recubierta de Cinc (Galvanizada).

[4] H. Asgari, M.R. Toroghinejad, M.A Golozar, "Relationship between (00.2) and (20.1) texture components and corrosion resistance of hot-dip galvanized cinc coatings". Journal of Materials Processing Technology, 198 (1-3), 54-59. 3 de marzo de 2008.

[5] C. A. Arias Maldonado, Estudio del comportamiento de un ambiente corrosivo con cloruros y sulfatos sobre una lámina de acero galvanizado. Bucaramanga, 2011. Trabajo de grado (Ingeniería Metalúrgica y Ciencia de Materiales). Universidad Industrial de Santander. Facultad de Fisicoquímicas.

[6] D. Sierra F., L. A. Quintero, Estudio del comportamiento de la corrosión de un acero galvanizado, en un ambiente que contiene cloruros y sulfatos mediante técnicas electroquímicas. Bucaramanga, 2012. Trabajo de grado (Ingeniería Metalúrgica y Ciencia de Materiales). Universidad Industrial de Santander. Facultad de Fisicoquímicas.

[7]. H. Asgari, M.R. Toroghinejad, M.A. Golozar. "Effect of coating thickness on modifying the texture and corrosion performance of hot-dip galvanized coatings". Current Applied Physics, 9(1), 59-66, junio de 2009.

[8] V. Padilla, P. Ghods, A. Alfantazi. "Corrosion film breakdown of galvanized steel in sulphatechloride solutions". Construction and building materials, 66, 447-457, 15 de septiembre de 2014.

[9] J. G. Castaño, C. A. Botero y S. Peñaranda. "Corrosión atmosférica del cinc en ambientes exteriores e interiores". Revista de metalurgia, 43(2), 133-145, marzo-abril de 2007.

[10] G. K. Mandal, D. Mandal, S. K. Das, R. Balasubramaniam and S.P. Mehrotra. "Microstructural study of galvanized coatings formed in pure as well as commercial grade Cinc baths". Transactions of the Indian Institute of Metals. 62(1), 35-40, February 2009.

[11] Asociación Latinoamericana de Cinc-LATIZA-. GalvinfoNotes, El rol del aluminio en el galvanizado continuo por inmersión en caliente, Junio de 2007.

[12] Y. Rico Oviedo, "Estructura y caracterización de los recubrimientos galvanizados por inmersión en caliente sobre aceros". Universidad Nacional Experimental Politécnica Antonio José de Sucre. Revista Digital de Investigación y Postgrado, 2(5), 368-378, octubre de 2012.

[13] P. R. Seré, J. D. Culcasi, C.I. Elsner y A.R. Di Sarli, "Factores que afectan a la estructura de los recubrimientos de cinc obtenidos por inmersión". Revista de Metalurgia, 33, (6), 376-381, 1997. 